\title{
SENSOR FAULT DETECTION AND IDENTIFICATION IN A PILOT PLANT UNDER PROCESS CONTROL
}

\author{
P. A. F. N. A. AFONSO, J. M. L. FERREIRA and J. A. A. M. CASTRO \\ Department of Chemical Engineering, University of Coimbra, Portugal
}

\begin{abstract}
7 he experimental evaluation of an automatic procedure for sensor fault detection and identification in a real process under closed-loop control is the objective of the present research. The scheme proposed here is very robust to faults in the main sensors of a multiloop control system, thus improving safety and reliability of plant operations. A state variable transformation is carried out in order to derive a model suitable for Recursive Least Squares (RLS) identification valid for all regimes of operation. The fault detection method is based on a moving window statistical analysis of the estimated model parameters. Simultaneously, a state estimation scheme, based on the Extended Kalman Filter (EKF), enables the fault identification, reduces false alarms and provides redundant measurements for alternative control purposes. Experimental runs were carried out in an industrial-scale pilot plant. Despite the large number of uncertainties and nonlinearities in the process, the system exhibited a good performance when faults occurred in the sensors of the control loops.
\end{abstract}

Keywords: fault detection; fault identification; fault tolerant control systems; industrial control

\section{INTRODUCTION}

The chemical process industries are characterized by a large diversity and complexity of plants that often depend on human operators, supported by some kind of automatic control system. Such a strategy is strongly dependent on the algorithms used to control the several sub-systems that cause a loss of generality in relation to the global process. In addition, there is a high degree of confidence in the information that flows through all kinds of instrumentation. A failure in the measuring device of a control loop can induce the controller to perform a set of undesirable and inappropriate control actions.

In order to ensure the safety of the plant and to improve the reliability of its operation, it is necessary to develop mechanisms that, in the case of a failure in the instrumentation, allow the detection and the identification of the fault, thus enhancing the robustness of the process control structure.

One of the most common schemes used to achieve this purpose is based on redundant systems which rely on multiple sensors to measure the same process variable. Another strategy with a great acceptance in industry is to verify if the value of a particular variable lies between acceptable limits, or to look at the highest variation observed between two successive measurements. More sophisticated techniques have, however, been proposed which make use of knowledge-based systems ${ }^{1}$ and of artificial neural networks ${ }^{2,3}$.

If a reasonably accurate model of the plant is available or if it can be obtained by identification then analytical detection techniques can be employed ${ }^{4}$. Several surveys concerned with different approaches to the problem of fault detection using process models have appeared. Willsky ${ }^{5}$ is the author of a classic paper discussing the most important techniques available at the time and more recently Isermann ${ }^{6}$, Patton ${ }^{7,8}$ and Frank ${ }^{9}$ have also focused on this subject.

A great variety of fault detection schemes rely on residual generation and testing, such as: the parity space approach ${ }^{10,11}$, dedicated observer schemes and the innovation-based approach ${ }^{7,12}$, as well as the parameter identification approach ${ }^{6,13,14}$. These Fault Detection and Identification (FDI) strategies focused on examples from the chemical and aerospace industries. However, most of the applications described in the literature were mainly tested in a simulated environment where many practical realities are totally absent. Moreover, they did not attempt to go further in what concerns the establishment of efficient control policies, particularly when a fault occurs. This is particularly important in the case where state and parameter estimation techniques are used for fault detection and identification.

This paper deals with the experimental implementation of an automatic scheme for fault detection and identification in an industrial-scale plant under multiloop SISO PI control. The main goal is to evaluate the behaviour of the system under feedback control when a failure occurs in any of the sensors measuring the controlled variables. This task was accomplished by making use of a dynamic model of the process whose parameters are time varying and not well known. Although the problem of multiple simultaneous faults has already been addressed ${ }^{3}$ the present work is based on the single-fault assumption. 


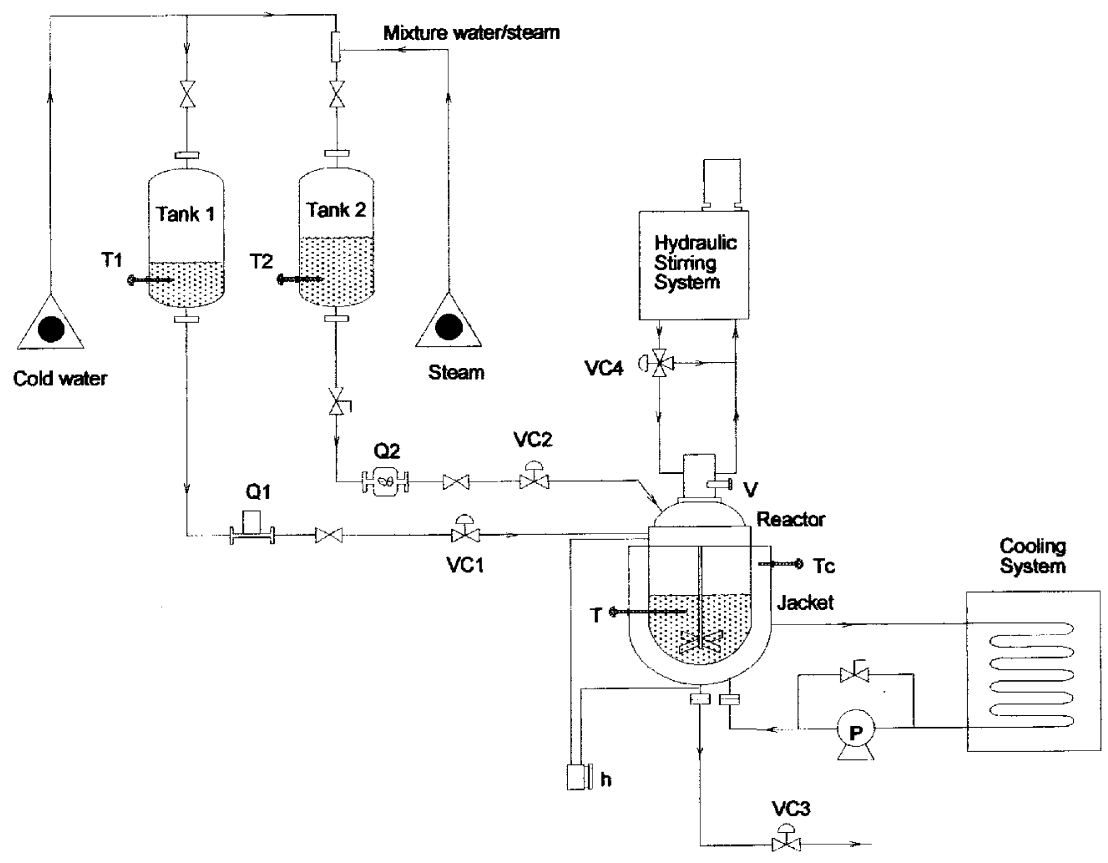

Figure 1. Simplified flowsheet of the pilot plant.

The on-line identification of the process model parameters was carried out by means of a variant of the basic Recursive Least Squares (RLS) algorithm. Since the original model is non-linear, a new set of auxiliary variables was defined in order to obtain a linear representation of the process. This is particularly useful in processes where variables suffer large variations, such as the case of batch processing plants.

The fault detection step is achieved through a statistical decision algorithm that analyses the behaviour of the process parameters provided by RLS. Simultaneously, a state estimation algorithm based on the Extended Kalman Filter (EKF) enables the identification of the fault by comparing the estimated values of the variables with the respective sensor measurements. Thus when a failure is detected and identified, the corresponding sensor is automatically disabled and control of the related process variable is carried out using an estimate supplied by EKF.

\section{PROCESS DESCRIPTION AND MODELLING}

The plant where the experimental tests were performed is depicted in Figure 1 and is similar to that proposed by Kershenbaum and Kittisupakorn ${ }^{15}$, for experimental testing of control algorithms. A nonadiabatic stirred tank reactor with a volume of 80 litres is fed by two liquid streams that come from two pressurized tanks. Cold water flows from tank 1 with flowrate $Q_{1}$ and temperature $T_{1}$ and hot water comes from tank 2 at temperature $T_{2}$ and flowrate $Q_{2}$. These flowrates are manipulated by control valves $\mathrm{VC}_{1}$ and $\mathrm{VC}_{2}$, respectively.

The coolant flowing through the jacket at temperature $T c$ provides partial cooling of the reactor contents which is at temperature $T$. The reactor outflow is governed by gravity and by control valve $\mathrm{VC}_{3}$ draining through a pipe into atmospheric pressure.

The level in the reactor as well as in the feeding tanks is measured by DP cells and all temperatures by Pt100 sensors. A mass flowmeter is employed to indicate flowrate $Q_{1}$ while $Q_{2}$ is measured by a mechanical volumetric flowmeter. All control valves $\mathrm{VC}_{1}, \mathrm{VC}_{2}, \mathrm{VC}_{3}$, and $\mathrm{VC}_{4}$ exhibit hysteresis and are operated by proportional electrical actuators.

Both data acquisition and actuation are carried out by a slave personal computer (equipped with 12-bit ADC/DAC devices). The process is under the supervision of a UNIX based workstation where the process status is graphically monitored in real time by using standard computer graphic techniques. More details can be seen elsewhere. ${ }^{16}$

Figure 2 shows the control loops and the corresponding input and output variables. Feedback controllers $\mathrm{C}_{1}, \mathrm{C}_{2}$, and

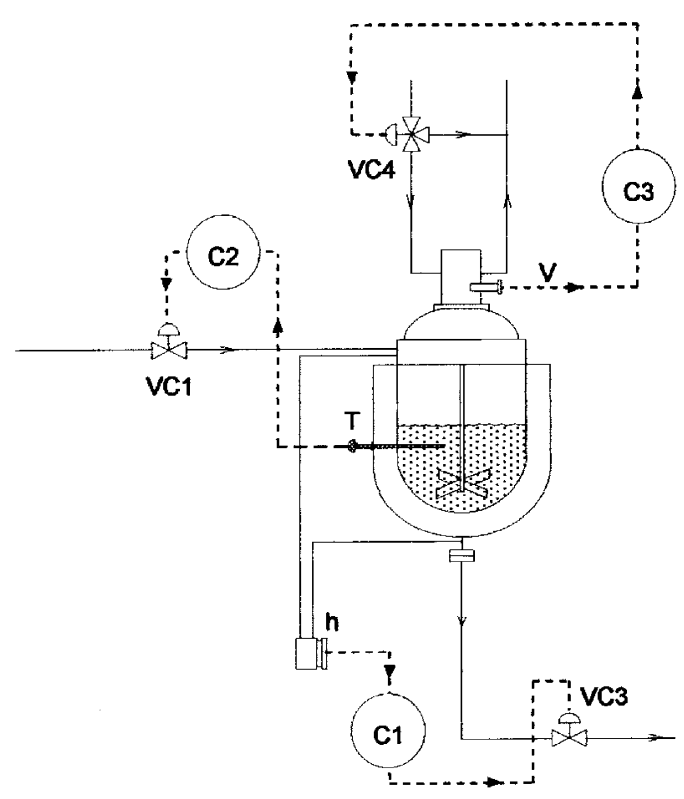

Figure 2. Pilot plant SISO multiloop control scheme. 
$\mathrm{C}_{3}$, are discrete versions of conventional PI controllers with a sampling period of $0.1 \mathrm{~min} . \mathrm{C}_{1}$ controls liquid level by manipulating the outlet flow through control valve $\mathrm{VC}_{3}$. Temperature inside the reactor is controlled with $\mathrm{C}_{2}$ that drives valve $\mathrm{VC}_{1}$ to regulate the cold stream flowrate $Q_{1}$. Finally, control valve $\mathrm{VC}_{4}$ is actuated by $\mathrm{C}_{3}$ in order to ensure a constant stirring speed.

In the absence of chemical reaction, a simple model can be derived based on total mass and energy balances. Assuming constant density, the total mass in the tank is given by:

$$
\frac{\mathrm{d} h}{\mathrm{~d} t}=\frac{1}{A}\left(Q_{1}+Q_{2}-Q_{3}\right)
$$

where the outlet flow $Q_{3}$ is a function of both reactor level and $\mathrm{VC}_{3}$ position $v_{3}$; this can be approximated by:

$$
Q_{3}=\beta \sqrt{h+h_{0}} \frac{\alpha v_{3}}{1+v_{3}(\alpha-1)}
$$

where $\alpha$ and $\beta$ are adjustable parameters. The dynamic behaviour of the reactor temperature can be obtained through the corresponding energy balance, assuming perfect mixing and uniform jacket temperature:

$$
\begin{aligned}
\frac{\mathrm{d} T}{\mathrm{~d} t}= & \frac{Q_{2}}{A h}\left(T_{2}-T\right)+\frac{Q_{1}}{A h}\left(T_{1}-T\right) \\
& +U_{g}(A+\pi \mathrm{d} r h) \frac{T c-T}{\rho C_{p} A h}
\end{aligned}
$$

Although the reactor is operated as a mixing tank, the corresponding model exhibits some mild nonlinearities associated with the state and input variables.

\section{SENSOR FAULT DETECTION AND IDENTIFICATION}

The strategy presented here for fault detection and identification is schematically shown in Figures 3(a) and (b), respectively. The fault detection method follows a

a) Fault detection stage

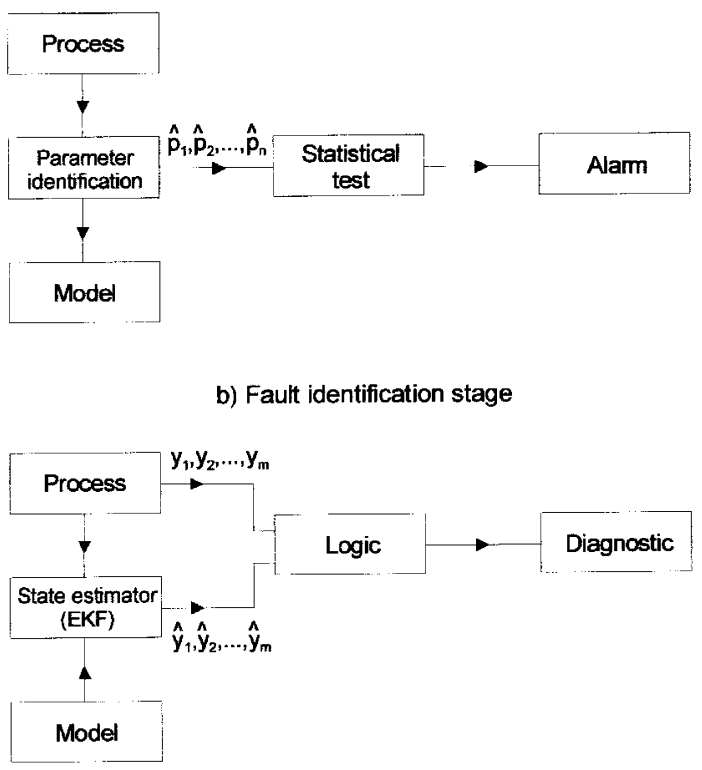

Figure 3. (a) Fault detection stage. (b) Fault identification stage. model based strategy, which includes the continuous updating of the process model parameters. The main goal is two-fold: first, to enable an advanced warning mechanism and second, to ensure good process representation for the fault identification and control stages. The warning mechanism, is achieved by following the changes in the estimated parameters and by presuming that unexpected variations are an indication of some kind of failure in the process. However, additional statistical analysis is required in order to launch a fault detection alarm from an observed change in the process parameters.

Figure 4 shows a schematic representation of the data flow through the instrumentation and control devices. The process control under normal operation is represented in Figure 4(a), and the strategy used in the case of a faulty situation is indicated in Figure 4(b).

In the present paper, RLS is employed with constant trace, as described by Shah and Cluett ${ }^{17}$, due to its relatively

\section{a) Normal operation}

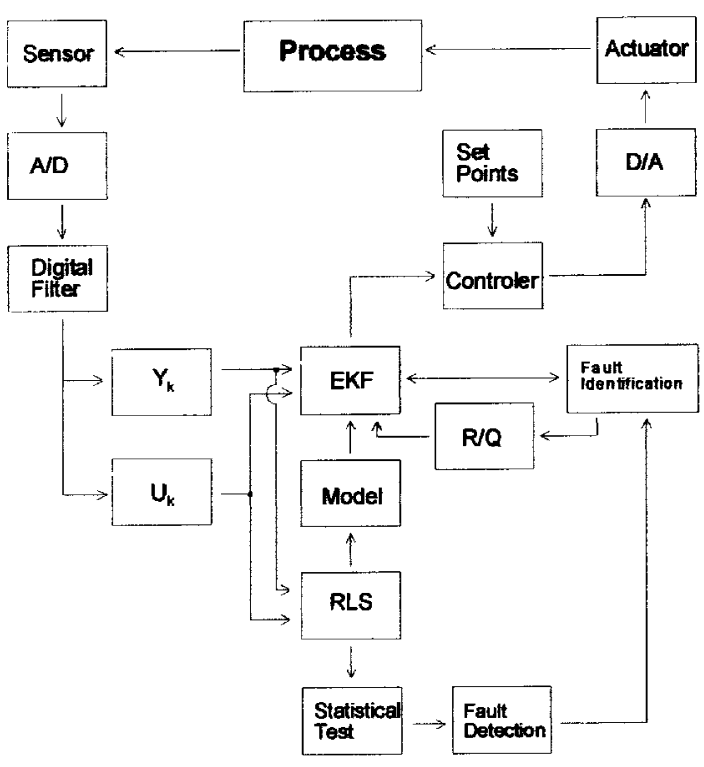

\section{b) Faulty operation}

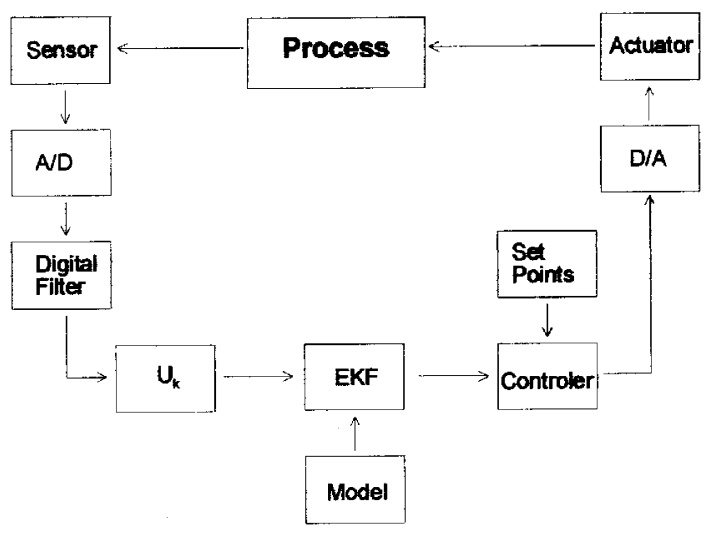

Figure 4. Normal and faulty operation flow paths (schematic diagram). 
fast convergence and numerical stability. It is a common policy to linearize the model equations, although the parameters would only be valid for small fluctuations of the process variables. Instead a different strategy has been chosen by defining a set of auxiliary variables (that combine two or more process variables) which make the system model globally linear with constant parameters. Equations (1) and (3) were discretized using a Euler first order approximation. After some reorganisation and defining the new auxiliary variables, the following relations are obtained:

$$
\begin{aligned}
y_{1, k+1}= & p_{1} y_{1, k}+p_{2} u_{1, k}+p_{3} u_{2, k}+p_{4} x_{1, k} \\
y_{2, k+1}= & p_{5} y_{2, k}+p_{6} x_{2, k}+p_{7} x_{3, k}+p_{8} x_{4, k}+p_{9} x_{5, k} \\
& +p_{10} x_{6, k}+p_{11} u_{3, k}+p_{12} x_{7, k}
\end{aligned}
$$

with the model parameters defined as:

$$
\begin{array}{ll}
p=1 & p_{10}=\frac{\Delta t U_{g}}{\rho C_{p}} \\
p_{2}=p_{3}=p_{4}=\frac{\Delta t}{A} & p_{11}=\frac{\Delta t U_{g} \pi \mathrm{d} r}{\rho C_{p} A} \\
p_{5}=1-\frac{\Delta t U_{g} \pi \mathrm{d} r}{\rho C_{p} A} & p_{12}=-p_{10} \\
p_{6}=-p_{7}=p_{8}=-p_{9}=\frac{\Delta t}{A} &
\end{array}
$$

and the following auxiliary variables:

$$
\begin{aligned}
& y_{1, k}=h_{k} \quad y_{2, k}=T_{k} \\
& x_{5, k}=\frac{Q_{1, k} T_{k}}{h_{k}} \\
& u_{1, k}=Q_{1, k} \\
& x_{2, k}=\frac{Q_{2 k} T_{2 k}}{h_{k}} \\
& x_{6, k}=\frac{T c_{k}}{h_{k}} \\
& u_{2, k}=Q_{2, k} \\
& x_{3, k}=\frac{Q_{2, k} T_{k}}{h_{k}} \\
& u_{3, k}=T c_{k} \\
& x_{1, k}=Q_{3, k} \\
& x_{4, k}=\frac{Q_{1, k} T_{1, k}}{h_{k}} \\
& x_{7, k}=\frac{T_{k}}{h_{k}}
\end{aligned}
$$

In order to quantify the efficiency of this procedure, the following definition is proposed

$$
\eta=\frac{F_{D}}{F_{V}}\left[1-\frac{F_{A}}{F_{A}+F_{D}}\right]
$$

where $F_{V}$ represents the number of real faults which occurred in the system and $F_{A}$ is the number of false alarms, i.e., the number of times a non existent fault was accepted. The number of real faults which were in fact detected is $F_{D}$. With this definition, both undetected faults and false alarms are penalized and therefore, $\eta$ will only have a value of 1 when all faults are detected (i.e. $F_{D}=F_{V}$ ) and no false alarms are produced (i.e., $F_{A}=0$ ).

The experimental tests revealed that this statistical analysis was not reliable enough to perform the fault identification step. The intrinsic noise of the measurements and the combination of variables used, unable the establishment of fault 'patterns', the so-called fault signature, often applied in fault identification? .

Alternatively, when a fault is detected, the estimated values of the state variables, given by the EKF, are compared to those supplied by the sensors. In this way, it is possible to validate the fault detection alarm and mainly to perform its identification, providing that multiple failures do not occur simultaneously. From the moment this validation is done, the estimation of the process parameters is suspended until the faulty sensor is replaced.

\section{EXPERIMENTAL RESULTS}

Despite the great interest in fault detection and identification schemes, very few practical applications have been reported in chemical industry plants under process control. This is why control strategies are often redefined in order to guarantee a safe plant operation. In the present work an attempt is made to address this problem.

Table 1 shows the values for the physical parameters of the process and the nominal operating conditions. Those signed with $\left(^{*}\right)$ were estimated by using an orthogonal distance regression for a set of 500 experimental points. The PI controller parameters were tuned accordingly to the Aström-Hägglund method ${ }^{19}$ and are shown in Table 2 as

Table 2. Controllers tuning parameters.

\begin{tabular}{llll}
\hline PI & Set-point & $k_{c}$ & $\tau_{i}$ \\
\hline $\mathrm{C}_{1}$ & $3 \mathrm{dm}$ & 5.5 & 80 \\
$\mathrm{C}_{2}$ & $29^{\circ} \mathrm{C}$ & 2.0 & 40 \\
$\mathrm{C}_{3}$ & $120 \mathrm{rpm}$ & 0.4 & 20 \\
\hline
\end{tabular}


Table 3. Initial parameter estimates.

\begin{tabular}{ll}
\hline$p_{1}=1$ & $p_{7}=-p_{2}$ \\
$p_{2}=5.68 \times 10^{-3}$ & $p_{8}=p_{2}$ \\
$p_{3}=p_{2}$ & $p_{9}=p_{2}$ \\
$p_{4}=-p_{2}$ & $p_{10}=2.32 \times 10^{-2}$ \\
$p_{5}=9.80 \times 10^{-1}$ & $p_{11}=2.00 \times 10^{-2}$ \\
$p_{6}=p_{2}$ & $p_{12}=-p_{10}$ \\
\hline
\end{tabular}

well as the plant operation set-points. The initial values of the parameter estimates used in the RLS are indicated in Table 3.

The methodology described above has been fully assessed by means of a large number of experimental runs. In most cases, these last for several hours and all included a starting-up period of 30 minutes. During this setup stage, the level control loop was under set-point and load excitation to enable an adequate estimation of the model parameters from the given initial values.

Based on all past experimental runs, the sample sizes $N_{1}$ and $N_{2}$ in the statistical decision test were investigated. It was found that the range $81 \leq N \leq 121$ minimizes false alarms, leading to very high levels of detection efficiency, as defined by equation (6). This is highlighted in Figure 5 where one can also see that for values of $91 \leq N \leq 111$ all process faults are detected and no false alarms are generated. In all subsequent experimental runs a value of $N=101$ was used.

For the sake of simplicity only a test run is selected for presentation here. Faults of different amplitudes and durations were artificially imposed in the level and temperature sensors. This is indicated in Table 4, where faults are expressed in terms of percentage in relation to the normal operating point. Information concerning the faulty sensor and the respective duration period is also included.

Figure 6 shows the results obtained in the plant when this set of faults was exerted in the level and temperature sensors in the PI control loops, $C_{1}$ and $C_{2}$ respectively, for a $700 \mathrm{~min}$ long test. In Figures 6(a) and (b), $h$ (Sensor) and $T$ (Sensor) represent the level and temperature as given by the corresponding measuring devices. It is also indicated the result of the EKF estimation, $h(E K F)$ and $T(E K F)$. The curves $h($ Real $)$ and $T$ (Real) identify the real values of the

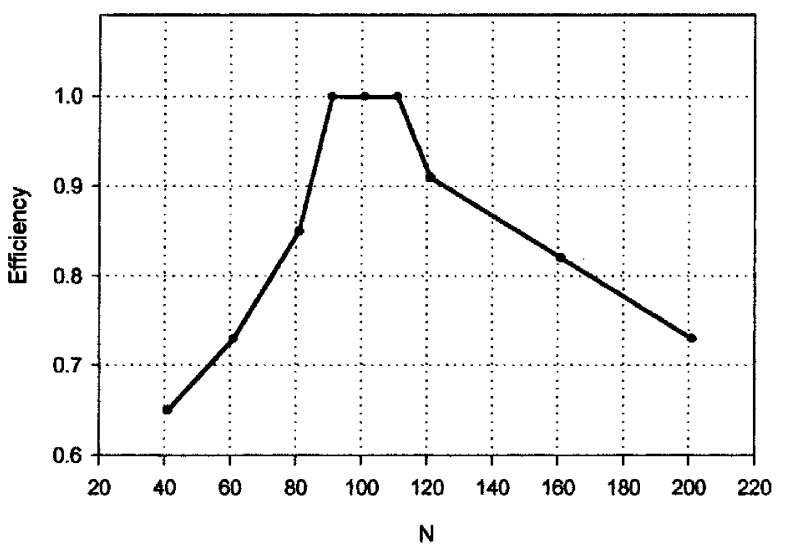

Figure 5. Statistical fault detection efficiency.
Table 4. Faults imposed in the process plant.

\begin{tabular}{lcc}
\hline Sensor & Amplitude & Time intervals (min) \\
\hline$h$ & $-33 \%$ & $75-80$ \\
$T$ & $+25 \%$ & $105-110$ \\
$h$ & $+15 \%$ & $145-165$ \\
$T$ & $-20 \%$ & $200-240$ \\
$h$ & $+30 \%$ & $270-300$ \\
$T$ & $-30 \%$ & $330-360$ \\
$h$ & $-30 \%$ & $390-420$ \\
$h$ & $+40 \%$ & $450-480$ \\
$T$ & $-50 \%$ & $530-560$ \\
$T$ & $-50 \%$ & $600-630$ \\
$T$ & $+20 \%$ & $660-690$ \\
\hline
\end{tabular}

process variables, i.e., the real level and temperature in the plant even when a sensor is disturbed. Figure 6(c) shows the results of $C_{1}$ and $C_{2}$ control actions, respectively control valve opening $v_{3}$ and $v_{1}$. Figures 7 (a) and (b), illustrate the behaviour of the remaining input variables: flowrates $Q_{1}$ and $Q_{2}$, control valve opening $v_{2}$ and temperatures $T_{1}, T_{2}$, and Tc.

The first fault took place (see Figure 6(a)) in the level sensor $75 \mathrm{~min}$ after the beginning of the operation and approximately $45 \mathrm{~min}$ after the above mentioned start-up period. Its effect can be directly observed by a rapid response of $C_{1}$ which quickly manipulates $v_{3}$ to compensate for this new situation, although in an undesirable fashion. The onus for this wrong behaviour of the controller lies with conventional feedback control schemes where the controller is completely dependent on the sensor. Meanwhile, as it is also apparent in Figure 8(a), the model parameter estimates $p_{2}$ and $p_{3}$ of level equation (4) suffer a sudden change. Although this might be meaningless by itself, with regards to a fault occurrence, the detection mechanism warns that the threshold limits for these parameters have been exceeded in the statistical decision test. This is outlined in Figures 9(a) and (b) where these limits of $F_{0}$ are also indicated by horizontal lines for a significance level of $\alpha=2 \%$ and corresponding to an alarm situation. In the present study, a value 5 times greater $\left(F_{0}<1 / 5 F_{0, \min }\right.$ and $\left.F_{0}>5 F_{0 \text { max }}\right)$ is assumed to indicate that an abnormal variation in the process parameters has occurred and, therefore, that a faulty situation exists in the system.

As soon as the fault is detected, the RLS estimation is halted and the fault identification stage is launched. This is achieved by comparing the values given by the sensors to those provided by EKF which is kept operating all the time. It is worth mentioning that this step alone cannot be used for detection because an occasional discrepancy between the real measurement and the EKF estimate does not mean that the process has suffered a 'structural' change. By comparing $h($ Sensor $)$ to $h(E K F)$ and $T$ (Sensor) to $T(E K F)$, see Figure 6(a), it is easily concluded that the level sensor was the faulty one. This sensor is immediately disabled and its measurement signal, usually sent to the controller, is then replaced by the corresponding EKF estimate. In few sampling times, the controller is able to quickly recover from this upset thus avoiding any further disruption of the system. In this case a 5 min long fault shows the behaviour of the process plant.

The success of this strategy, and particularly the control system performance, clearly relies upon the way the EKF is used and upon the process model. With respect to the first, 

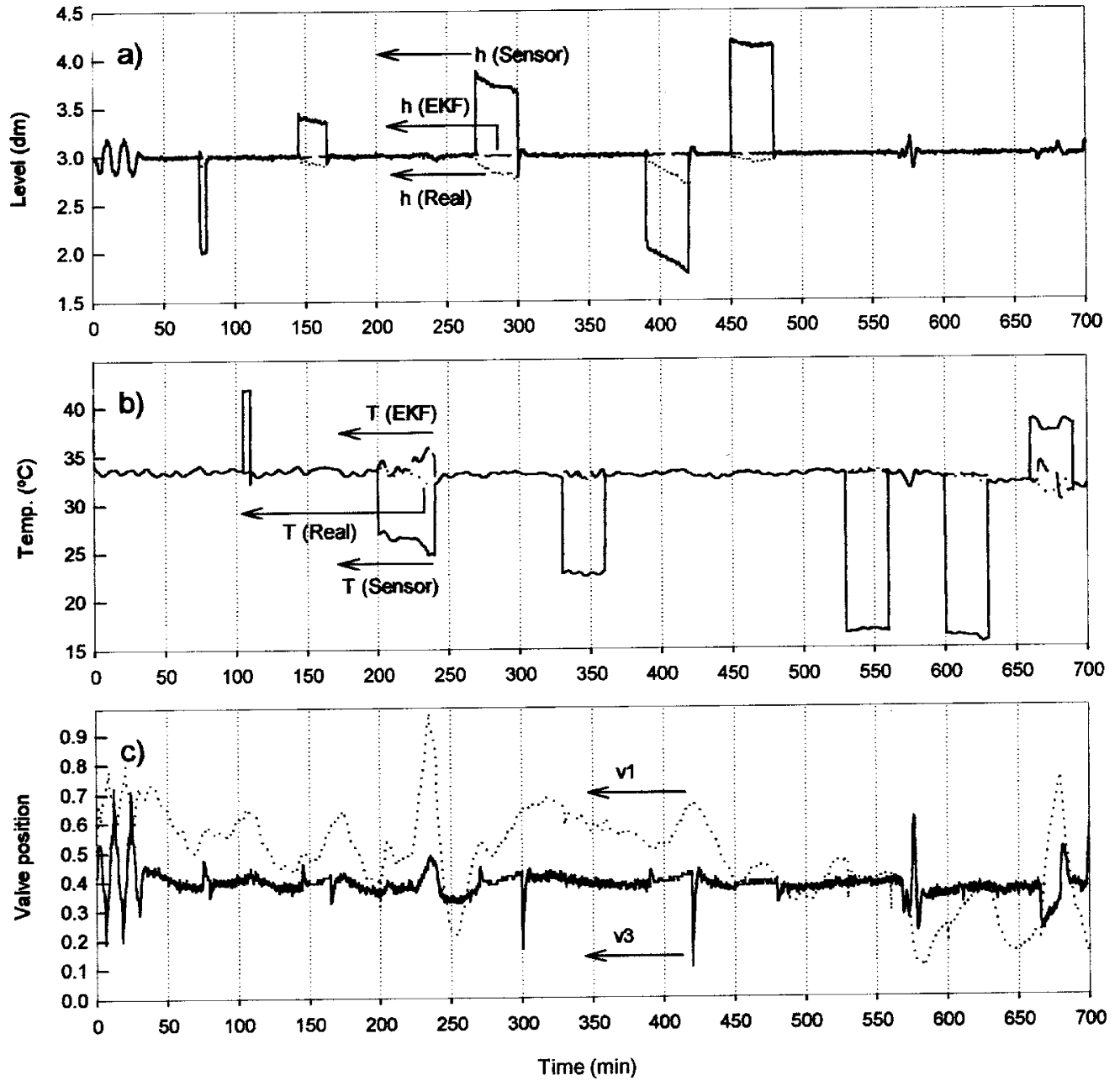

Figure 6. Evolution of process variables in the pilot plant where several faults were introduced in level and temperature sensors.
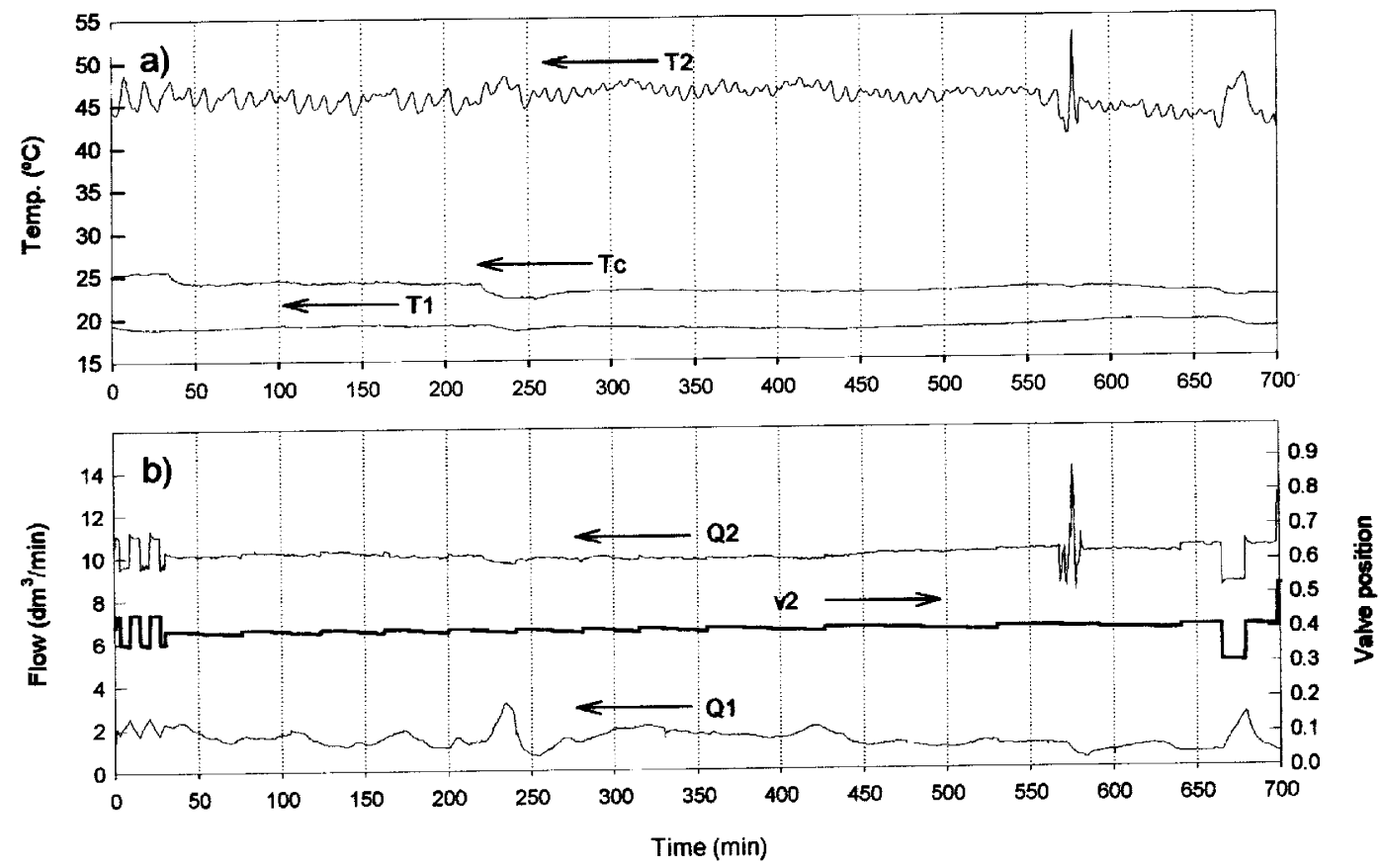

Figure 7. Additional process variable information described in Figure 6. 

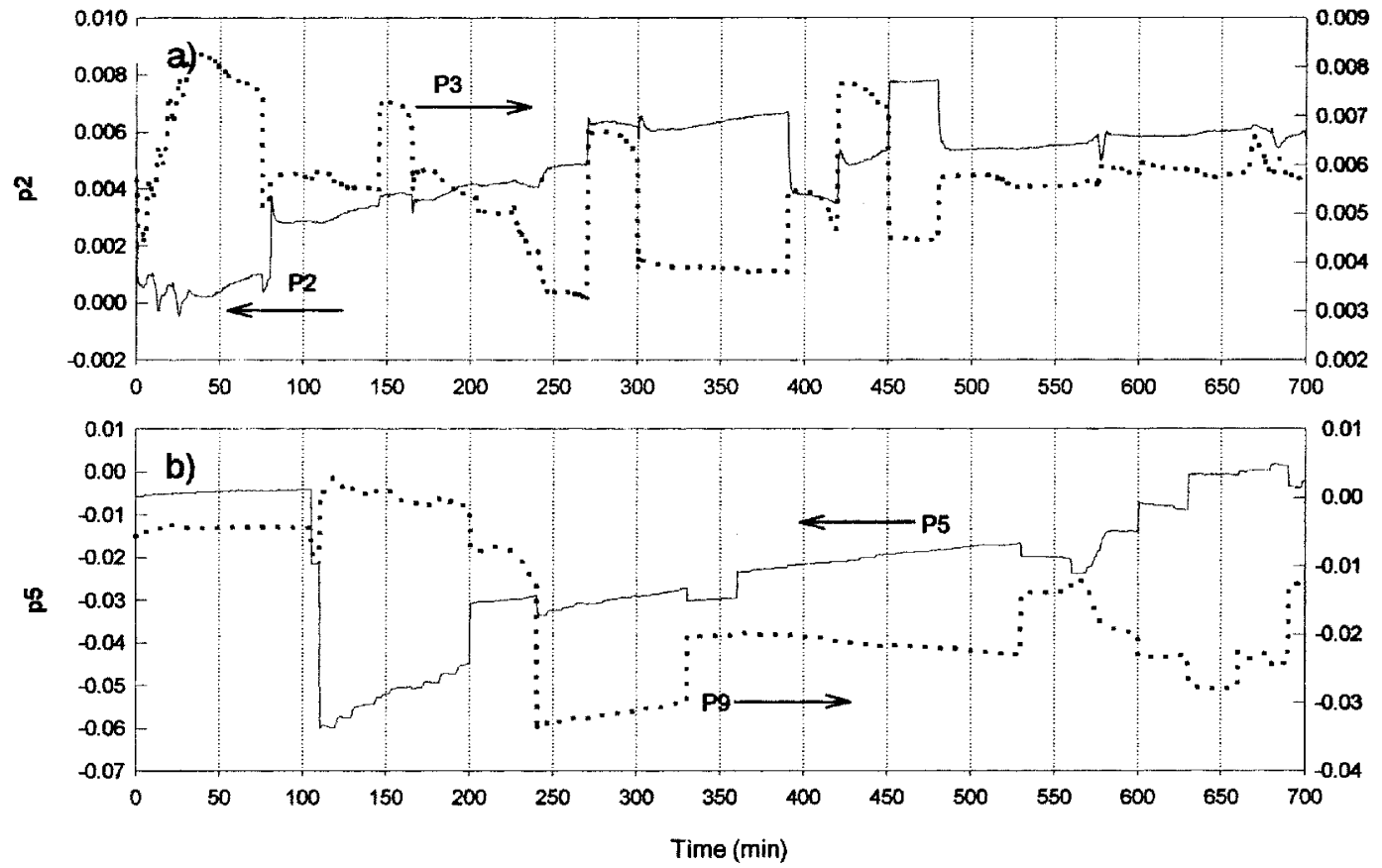

Figure 8. Estimated process parameters with RLS. Parameters p2, p3 and p5, p9 are indicated in graphics (a) and (b) respectively.

the procedure is the following: during the 'normal' period, the EKF is made to believe both in the model and in the observations, i.e., the values of model and measurement noise covariance matrices, $\mathbf{Q}$ and $\mathbf{R}$, are of the same order of magnitude. As soon as a fault is detected, i.e., during the 'abnormal' period, the diagonal element of matrix $\mathbf{R}$, corresponding to the faulty sensor, is made few orders of magnitude higher. This is to force the state estimator to disregard only that observation and, therefore, to increase its confidence in the corresponding model prediction. This is also in accordance with the disablement of the faulty sensor for all purposes until it is replaced or repaired. During this period, the control system becomes quite dependent on the model and, therefore, it is strongly recommended to have an up to date and properly tuned process model. Since it is not advisable to proceed with the RLS parameter estimation in an abnormal situation the best set of parameters available up to the time the fault was detected is used thereafter.
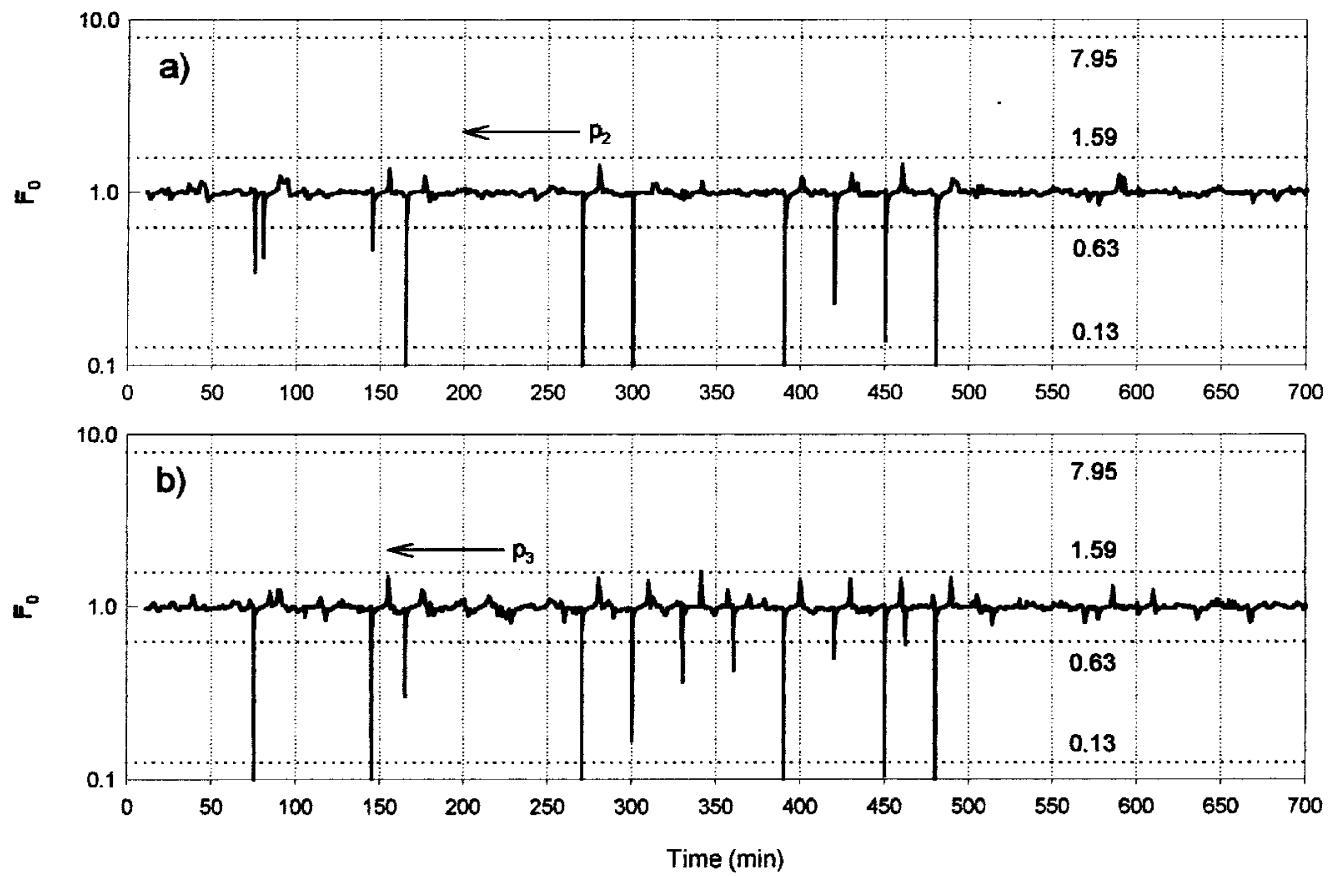

Figure 9. Statistical test corresponding to the parameters depicted in Figure 8(a). Parameter p2 is indicated in graphic (a) and p3 is indicated in graphic (b). 
A previously specified set of subsequent faults, of different amplitudes and durations, was applied to both level and temperature sensors, as described in Table 4. The results obtained in the pilot plant for faults in the temperature sensor are depicted in Figure 6(b). Globally, this means that, in a period of five to six hours, eleven faults were introduced into the system. The scheme presented here was able to detect all of them in spite of a small drift in two cases of the level control. This can be associated to the fact that a low precision flowmeter was used to measure flowrate, $Q_{2}$ of the main inlet stream (see Figure 7). In addition to this, the actuator of control valve $\mathrm{VC}_{3}$ is not sufficiently sensitive to carry out small corrective actions requested by the controller. Finally, the level measurement is affected by intense noise which arises from the strong agitation in the vessel. Due to problems in the water and steam supplies, two unexpected events occurred: one between 570 and $580 \mathrm{~min}$ and the other between 665 and $680 \mathrm{~min}$. Both arose from difficulties in the manual control of level in feeding tank 2, which in turn, are the result of strong oscillations in the pressure of the main water supply. It is worth mentioning that both events disturbed level and temperature in the reactor and that the second occurred while a fault was present in the system. In the first case, no false alarm was generated although the reactor level changed sharply.

Despite all of this, the performance of our FDI strategy is quite remarkable particularly in what concerns its efficiency (see equation (6)). Furthermore, the time required for detection (usually from two to three sampling times) and the ability to avoid drastic disturbances induced by the controllers as a result of the erroneous information from the sensors, demonstrates its capabilities for industrial application.

Figure 8 (b) shows the behaviour of the parameter estimates of $p_{5}$ and $p_{9}$ relative to the energy balance, equation (5), and their statistical analysis is represented in Figure 10(a) and (b). For simplicity, only this small set is represented because the profiles of other process parameters are analogous in similar situations. In fact some of them exhibit different degrees of sensitivity when faults occurred, but the most important issue is to stress the global effect on process parameters changes.

\section{CONCLUSIONS}

Several experimental runs have been carried out in an industrial scale pilot plant to test the scheme proposed for process control with failures in the sensors of the control loops. The results obtained are very promising considering that the process plant can operate very satisfactorily for periods longer than $30 \mathrm{~min}$ in the event of large faults in the sensors that drive the PI controllers. This is of much importance in industrial systems because persistent erratic data from faulty sensors can lead to wrong controller actions and thus to serious and irreversible damage to the process.

This FDI strategy can validate the information that flows through the instrumentation and consequently improve plant reliability. It is based on the process dynamics and therefore requires a reasonably well tuned process model. However, its simplicity, numerical robustness and the small computer effort required, makes it amenable for small to medium sized industrial applications.

Despite the good results obtained in the case of faults in the sensors measuring the state variables used the main control loops, additional work is under way in other areas. That is the case of faults in the controller actuators and in the sensors outside the control loops. Other problems deserving further investigation are those concerned with the detection and the identification of slow varying faults, which are also of much industrial importance.
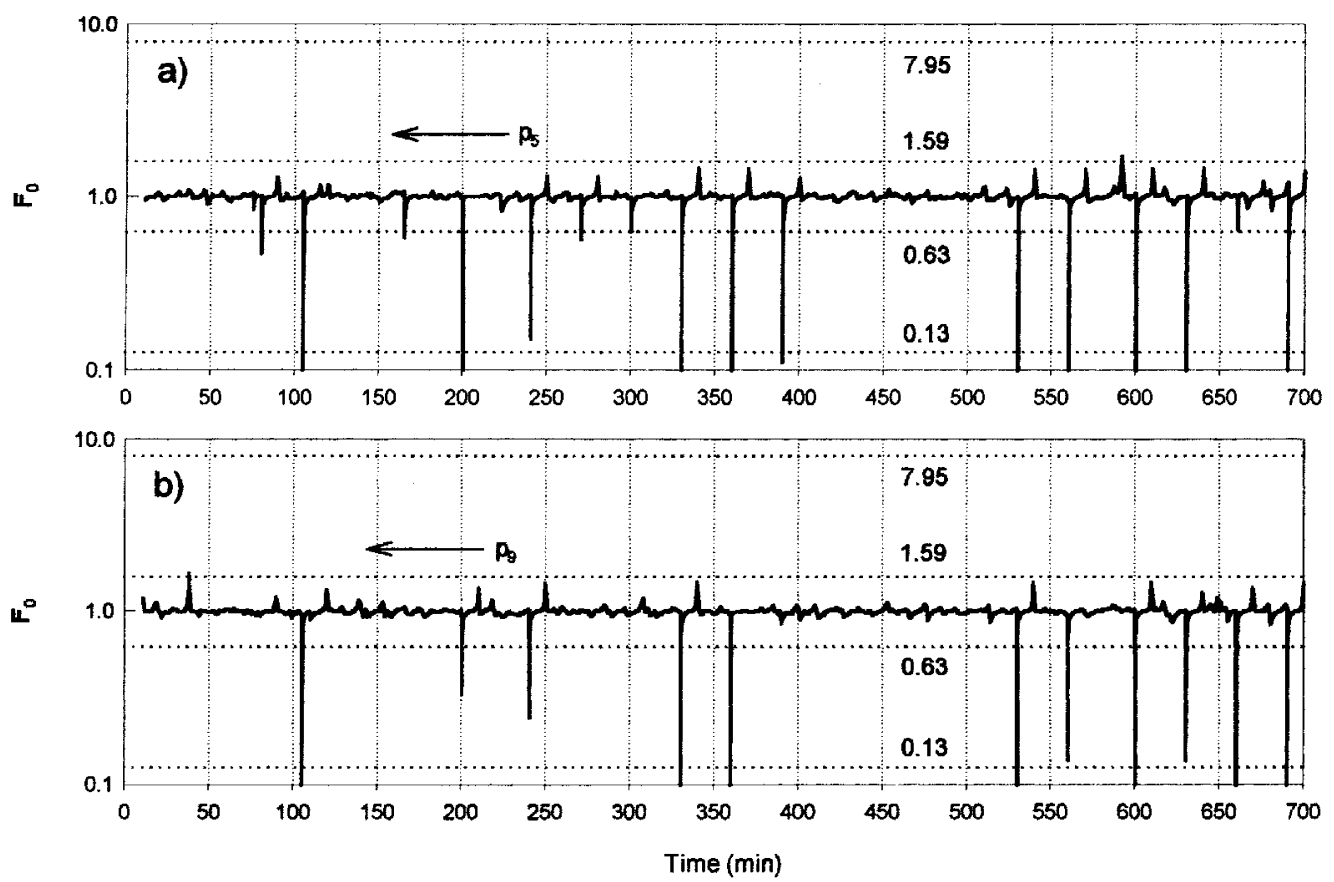

Figure 10. Statistical test corresponding to the parameters depicted in Figure 8(b). Parameter p5 is indicated in graphic (a) and p9 is indicated in graphic (b). 


\section{NOMENCLATUR E}

A cross-sectional area of the reactor

$C_{i} \quad$ PI controllers

$C_{p} \quad$ fluid heat capacity

$\mathrm{d} r \quad$ reactor diameter

$h \quad$ liquid level in the reactor

$h_{0} \quad$ level offset

$p_{i} \quad$ linear model parameter

$F_{D} \quad$ number of faults detected

$F_{V} \quad$ number of process faults

$F_{A} \quad$ number of false alarms

$Q_{1}, Q_{2}$ hot and cold water inlet flowrates

$\mathrm{O}_{3} \quad$ reactor outlet flowrate

$T$ fluid temperature in the reactor

$T_{i} \quad$ fluid temperature in tank $i$

Tc coolant temperature

$u_{i k} \quad$ input process variable

$U_{g} \quad$ global heat transfer coefficient

$v_{i} \quad$ position of control valve $\mathrm{VC}_{i}$

$x_{i, k} \quad$ linear auxiliary variable that combines process variables

$Y_{i, k} \quad$ linear output process variable

\section{Greek letters}

$\alpha, \beta \quad$ valve constants

$\Delta t \quad$ sampling time

$\rho \quad$ fluid density

\section{ACKNOWLEDGEMENT}

Financial support from FLAD (Project P-534) and from STRIDEIndustry (Project STRD/TRP/0013) is gratefully acknowledged. This project has been partially supported by NARES-Natural Resins, Ltd. One of the authors, Mr P. Afonso, is also indebted to JNICT (BD/612/90-IB) for financial assistance.

\section{REFERENCES}

1. Rich, S. H. and Venkatasubramanian, V., 1987, Model-based reasoning in diagnostic expert systems for chemical process plants, Comput Chem Eng, 11: 111-120.

2. Venkatasubramian, V. and Chan, K., 1989, A neural network methodology for process fault diagnosis, AIChE J, 35: 1993-2000.

3. Watanabe, K., Hirota, S. H., Hou, L. and Himmelblau, D. M., 1994, Diagnosis of multiple simultaneous fault via hierarchical artificial neural networks, AIChE J, 40: 839-848.

4. Chang, C. T., Mah, K. N. and Tsai, C. S., 1993, A simple design strategy for fault monitoring systems, AIChE J, 39: 1146-1163.
5. Willsky, A. S., 1976, A survey of design methods for failure detection in dynamic systems, Automatica, 12: 601-611.

6. Isermann, R., 1984, Process fault detection based on modelling and estimation methods survey, Automatica, 20: 387-404.

7. Patton, R. J., Frank, P. and Clark, R. (Eds.), 1989, Fault Diagnosis in Dynamic Systems - Theory and Applications, (Prentice-Hall, NJ).

8. Patton, R. J., 1994, Robust model-based fault diagnosis: The state of the art, Proc IFAC Symp SAFEPROCESS'94, Espoo, June 13-16, 124.

9. Frank, P. M., 1990, Fault diagnosis in dynamic systems using analytical and knowledge-based redundancy-A survey and new results, Automatica, 26: 459-474.

10. Chow, E. Y. and Willsky, A. S., 1984, Analytical redundancy and the design of robust failure detection system, IEEE Trans Aerospace Electron Sys, 14: 558-563.

11. Gertler, J. and Luo, Q., 1989, Robust isolable models for failure diagnosis, AIChE J, 35: 1856-1868.

12. Clark, R. N., Fosth, D. C. and Walton, V. M., 1975, Detection instrument malfunctions in control systems, IEEE Trans Aerospace Electron Sys, 11: 465-473.

13. Bloch, G., Ouladsine, M. and Thomas, P., 1995, On-line fault diagnosis of dynamic systems via robust parameter estimation, Control Eng Practice, 3(12): 1709-1717.

14. Hofling, T. and Isermann, R., 1996, Fault detection based on adaptive parity equations and single-parameter tracking, Control Eng Practice, 4(10): 1361-1369.

15. Kershenbaum, L. S. and Kittisupakorn, P., 1994, The use of a partially simulated exothermic (PARSEX) reactor for experimental testing of control algorithms, Trans IChemE, 72(A): 55-63.

16. Afonso, P. A. F. N. A., 1988, Sistemas de detecção e identificação de falhas na industria dos processor quimicos, PhD Thesis, (University of Coimbra, Portugal).

17. Shah, S. L. and Cluett, W., 1991, Recursive least squares based estimation schemes for self-tuning control, Can J Chem Eng, 69: 89-96.

18. Montgomery, D. C., 1991, Introduction to Statistical Quality Control, $2^{\text {nd }}$ ed, (John Wiley \& Sons, NY).

19. Aström, K. J. and Hägglund, I., 1984, Automatic tuning of simple regulators with specification on phase and amplitude margins, Automatica, 20: 465-651.

\section{ADDRESS}

Correspondence concerning this paper should be addressed to Professor J. Castro, Department of Chemical Engineering, University of Coimbra, 3000 Coimbra, Portugal.

The manuscript was received 11 July 1997 and accepted for publication after revision 6 April 1998. 